\title{
A quantitative high resolution voxel-wise assessment of myocardial blood flow from contrast-enhanced first-pass magnetic resonance perfusion imaging: microsphere validation in a magnetic resonance compatible free beating explanted pig heart model
}

\author{
Andreas Schuster 1,2*, Matthew Sinclair'1, Niloufar Zarinabad', Masaki Ishida', \\ Jeroen P.H.M. van den Wijngaard ${ }^{3}$, Matthias Paul ${ }^{1}$, Pepijn van Horssen ${ }^{3}$, \\ Shazia T. Hussain ${ }^{1}$, Divaka Perera ${ }^{1,4}$, Tobias Schaeffter ${ }^{1}$, Jos A.E. Spaan ${ }^{3}$, \\ Maria Siebes ${ }^{3}$, Eike Nagel ${ }^{1,5}$, and Amedeo Chiribiri ${ }^{1}$
}

\begin{abstract}
${ }^{1}$ Division of Imaging Sciences and Biomedical Engineering, King's College London British Heart Foundation (BHF) Centre of Excellence, National Institute of Health Research (NIHR) Biomedical Research Centre at Guy's and St. Thomas' NHS Foundation Trust, Wellcome Trust and Engineering and Physical Sciences Research Council (EPSRC) Medical Engineering Centre, The Rayne Institute, St. Thomas' Hospital, Lambeth Palace Road, London, UK; ${ }^{2}$ Department of Cardiology and Pneumology and German Centre for Cardiovascular Research (DZHK, Partner Site Göttingen), Georg-August-University, Göttingen, Germany; ${ }^{3}$ Department of Biomedical Engineering and Physics, Academic Medical Centre, Amsterdam, The Netherlands; ${ }^{4}$ King's College London BHF Centre of Excellence, NIHR Biomedical Research Centre and Department of Cardiology, Guy's and St. Thomas' NHS Foundation Trust, London, UK; and ${ }^{5}$ Division of Cardiovascular Imaging, Goethe University Frankfurt and German Centre for Cardiovascular Research (DZHK, Partner Site Rhine-Main), Frankfurt, Germany
\end{abstract}

Received 5 November 2014; accepted after revision 30 January 2015; online publish-ahead-of-print 26 March 2015

Aims

To assess the feasibility of high-resolution quantitative cardiovascular magnetic resonance (CMR) voxel-wise perfusion imaging using clinical 1.5 and $3 \mathrm{~T}$ sequences and to validate it using fluorescently labelled microspheres in combination with a state of the art imaging cryomicrotome in a novel, isolated blood-perfused MR-compatible free beating pig heart model without respiratory motion.

Methods MR perfusion imaging was performed in pig hearts at $1.5(n=4)$ and $3 T(n=4)$. Images were acquired at physiological and results flow ('rest'), reduced flow ('ischaemia'), and during adenosine-induced hyperaemia ('stress') in control and coronary occlusion conditions. Fluorescently labelled microspheres and known coronary myocardial blood flow represented the reference standards for quantitative perfusion validation. For the comparison with microspheres, the LV was divided into 48 segments based on a subdivision of the 16 AHA segments into subendocardial, midmyocardial, and subepicardial subsegments. Perfusion quantification of the time-signal intensity curves was performed using a Fermi function deconvolution. High-resolution quantitative voxel-wise perfusion assessment was able to distinguish between occluded and remote myocar$\operatorname{dium}(P<0.001)$ and between rest, ischaemia, and stress perfusion conditions at $1.5 \mathrm{~T}(P<0.001)$ and at $3 \mathrm{~T}(P<0.001)$. CMR-MBF estimates correlated well with the microspheres at the AHA segmental level at $1.5 \mathrm{~T}(r=0.94, P<0.001)$ and at $3 \mathrm{~T}(r=0.96, P<0.001)$ and at the subendocardial, midmyocardial, and subepicardial level at $1.5 \mathrm{~T}(r=0.93$, $r=0.9, r=0.88, P<0.001$, respectively $)$ and at $3 \mathrm{~T}(r=0.91, r=0.95, r=0.84, P<0.001$, respectively).

Conclusion CMR-derived voxel-wise quantitative blood flow assessment is feasible and very accurate compared with microspheres. This technique is suitable for both clinically used field strengths and may provide the tools to assess extent and severity of myocardial ischaemia.

Keywords

cardiovascular magnetic resonance imaging • myocardial perfusion imaging • voxel-wise quantification • fermi deconvolution • microspheres • Isolated heart perfusion

* Corresponding author. Tel: +49 55139 20870; Fax: +49 55139 22026, E-mail: andreas_schuster@gmx.net

Published on behalf of the European Society of Cardiology. All rights reserved. (C) The Author 2015. For permissions please email: journals.permissions@oup.com. 


\section{Introduction}

Coronary artery disease (CAD), subsequent myocardial infarction, and heart failure of ischaemic origin are major determinants of mortality and morbidity. ${ }^{1}$ Myocardial ischaemia constitutes an important pathophysiological substrate ${ }^{2}$ and can be detected with cardiovascular magnetic resonance (CMR) perfusion imaging. ${ }^{3}$ CMR perfusion imaging compares favourably to other non-invasive methods ${ }^{4-6}$ and has established itself as an important non-invasive clinical test to guide patient management. ${ }^{7}$ Furthermore, it provides quantitative assessment of myocardial blood flow (MBF), ${ }^{8-10}$ and validation studies have shown excellent agreement with microspheres. $^{11-13}$ The validation of quantitative perfusion techniques has however been restricted to larger areas such as standard myocardial segments as proposed by the AHA to increase the signal-to-noise ratio. ${ }^{14}$ There is evidence that high-resolution perfusion techniques enhance the diagnostic accuracy for the detection of significant CAD predominantly by detecting more subendocardial ischaemia within standard segments. ${ }^{15,16}$ Furthermore, quantitative MBF assessment in patients and canines based on voxel-wise perfusion measurements has been demonstrated at 1.5 and $3 \mathrm{~T}^{17,18}$ However, there are assumptions inherent to the quantitative methodology involved such as estimation of MBF from the relationship of the arterial input function and the myocardial response curve, which can be influenced by cardiac output, ejection fraction, and heart rate.

We have recently described a novel magnetic resonance (MR) compatible explanted free beating pig heart model that allows precise control of regional blood flow and in combination with histological assessment of fluorescently labelled microspheres represents a suitable vehicle for quantitative perfusion validation. ${ }^{19}$

The aim of the current study was to assess the feasibility of highresolution quantitative CMR perfusion imaging based on voxel-wise data acquisition using clinical 1.5 and $3 \mathrm{~T}$ perfusion sequences, compare their accuracies and to validate them using fluorescently labelled microspheres in combination with a state of the art imaging cryomicrotome. $^{20,21}$

\section{Methods}

\section{Experimental design of the study}

All animal experiments were conducted after approval by the UK Home Office in accordance with the UK Animals (Scientific Procedures) Act of 1986 and in compliance with the World Medical Association Declaration of Helsinki regarding ethical conduct of research involving animals. Eight healthy Large White Cross Landrace pigs weighing between 43 and $54 \mathrm{~kg}$ were included in this study (Harlan Laboratories, UK). Hearts were harvested as previously described. ${ }^{19,22}$ Sedation was performed with ketamine $(10 \mathrm{mg} / \mathrm{kg}$ i.m.) and xylazine $(0.3 \mathrm{mg} / \mathrm{kg}$ i.m.) in combination with alphaxolone for general intravenous anaesthesia $(1.5 \mathrm{mg} / \mathrm{kg}$ i.v.). Heparin was administered (5000 IU) and exsanguination started through the superior vena cava. The hearts were removed after transection of the great heart vessels and perfused with intra-coronary infusion of cold $\left(4^{\circ} \mathrm{C}\right)$ cardioplegic solution (Martindale Pharmaceuticals, Romford, Essex, UK). After transport to our institution, catheters were inserted into the coronary arteries before reperfusion. The heart was subsequently connected to the perfusion circuit as illustrated in Figure 1. A detailed scheme of the perfusion setup is also provided elsewhere. ${ }^{19,22}$ To expand the left ventricular (LV) cavity, a pressure balloon was inserted through the aortic valve into the left ventricle and inflated to a pressure of $50 \mathrm{mmHg}$. After the hearts were cannulated, pressure-controlled perfusion of the coronary arteries was started at around $50 \mathrm{mmHg}$. Over $\sim 5$ min pressure was slowly increased to achieve a constant perfusion rate of $0.8 \mathrm{~mL} / \mathrm{min} / \mathrm{g}$. In the event of ventricular fibrillation, electrical defibrillation was performed. In case of persisting bradycardia, right atrial pacing was performed with an in house built MR compatible pacing wire. After preparation stability was achieved, the left anterior descending (LAD) coronary artery was occluded to create a territory with a perfusion defect and CMR imaging was started. The occlusion of the LAD allowed multiple measurements of abnormal and normal perfusion values in each heart. Perfusion-CMR was performed at rest, with $50 \%$ flow reduction and during pharmacological vasodilation with adenosine (Figure 2). During adenosine infusion coronary artery pressure dropped due to vasodilation. The pump flow was subsequently increased to restore the same coronary perfusion pressure as during the resting state, which resulted in vasodilation-induced hyperaemic blood flow.

\section{CMR perfusion imaging}

The animal studies were performed on $1.5 \mathrm{~T}$ (Achieva CV, Philips, Best, The Netherlands), $(n=4)$ and 3 T (Achieva TX, Philips Best, The Netherlands), $(n=4)$ clinical MR scanners. For signal reception, a clinical interventional $\mathrm{L}$ flex receiver coil array was tightly positioned around the heart chamber, which was then placed in the isocentre of the magnet. CMR perfusion images were acquired in short-axis orientation of the LV according to a recognized standard model. ${ }^{14}$

CMR perfusion imaging was performed at $1.5 \mathrm{~T}$ with a balanced turbo gradient echo pulse sequence accelerated with a three-fold k-t broad linear speed up technique (BLAST) using 11 training profiles yielding a typical spatial resolution of $1.9 \times 2 \times 10 \mathrm{~mm}$. TE/TR was 1.35/2.71; $50^{\circ}$ flip angle; $90^{\circ}$ saturation prepulse and $100 \mathrm{~ms}$ prepulse delay.

At $3 \mathrm{~T}$, we used a saturation recovery gradient echo pulse sequence accelerated with $\mathrm{k}-\mathrm{t}$ BLAST ( $\mathrm{k}$-t factor 5 and 11 training profiles) with a repetition time of $2.7 \mathrm{~ms}$, echo time of $0.9 \mathrm{~ms}$, flip angle $20^{\circ}$, spatial resolution at $1.3 \times 1.3 \times 8 \mathrm{~mm}$, and $120 \mathrm{~ms}$ saturation prepulse delay.

Perfusion-CMR was performed using a dual bolus scheme with $5 \mathrm{~mL}$ of dilute $(0.007 \mathrm{mmol} / \mathrm{mL})$ and $5 \mathrm{~mL}$ of neat $(0.07 \mathrm{mmol} / \mathrm{mL})$ gadobutrol bolus injections (Gadovist, Bayer Healthcare, Leverkusen, Germany). ${ }^{23}$ The contrast agent was injected into the arterial limb of the tubing that directly supplied the coronary arteries (Figure 1). The signal intensity (SI) time curves including arterial input function and myocardial response curves were obtained in the same imaging views as previously described. ${ }^{19}$ Post-processing of perfusion images was performed with a dedicated software (Viewforum Prototype, Version 7.2V1WIP1, Philips Healthcare). ${ }^{24}$

\section{Quantitative analysis of MR perfusion imaging}

Following motion correction and contour delineation, SI-time curves of the CMR perfusion images were extracted for each voxel and then incorporated into a Fermi function deconvolution algorithm for quantitative analysis $^{25}$ using MATLAB (Natick, MA, USA).

For comparison with microspheres, the $L V$ was divided into American Heart Association (AHA) standard segments. ${ }^{14}$ This was performed due to the characteristic microsphere deposition pattern in end-arteries ${ }^{26}$ which does not allow a validation at the voxel level and in line with previously published methodology. ${ }^{18}$

Each segment was subdivided into subendocardial, midmyocardial, and subepicardial subsegments to allow validation of regional quantitative perfusion estimates. Independent Sl-time curves based on 100 voxels per myocardial AHA segment were obtained and subjected to quantitative 


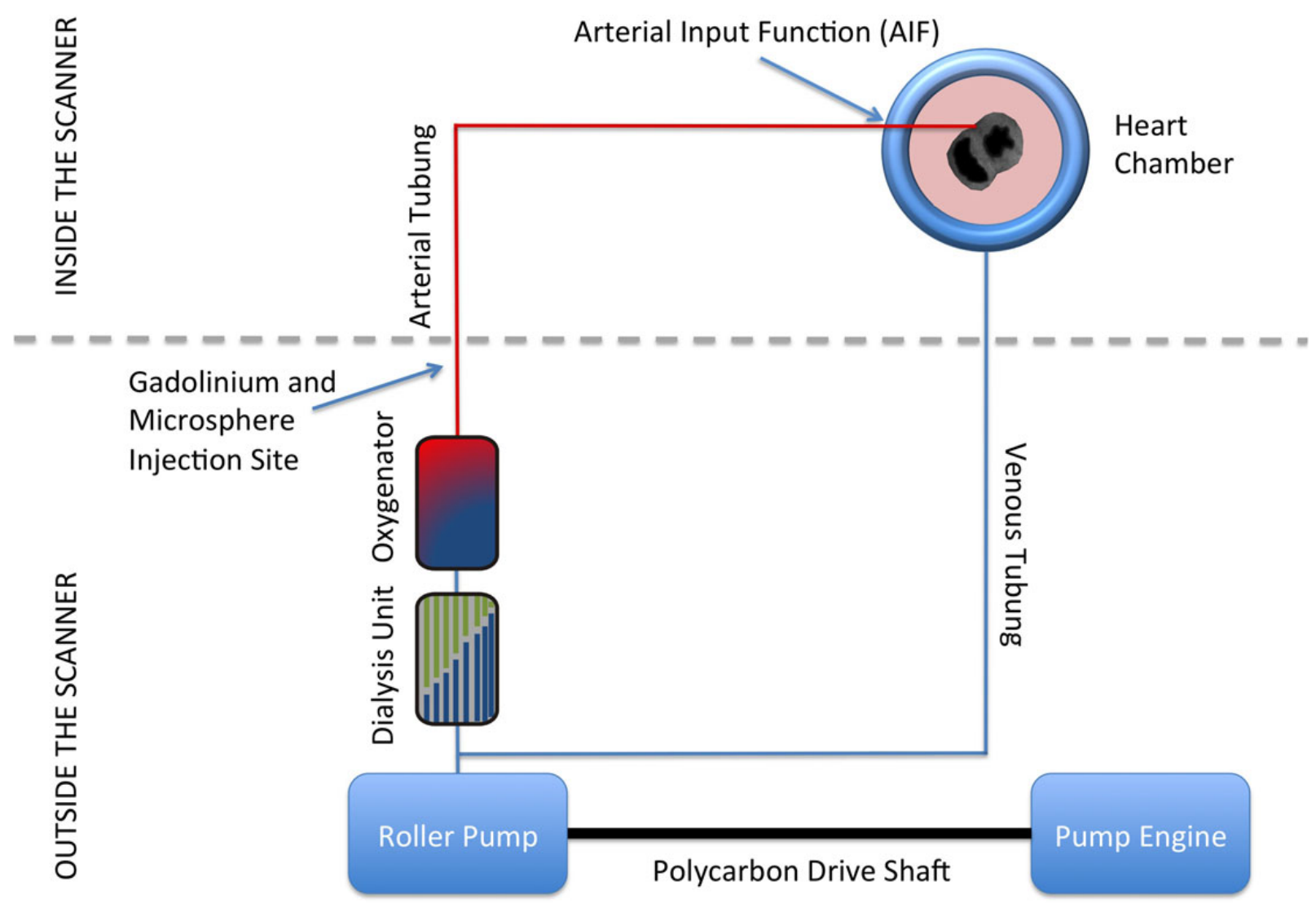

Figure I The figure provides a schematic view of the isolated perfused heart setup. The system is divided into a part that is located within the MR scanner and a part located outside the MR scanner. All electrical equipment is completely shielded and stored outside the five Gauss line. ${ }^{19,22}$ The blood is transported using a roller pump system through a dialysis module and then through a blood oxygenator with integrated heat exchanger. The oxygenated blood is then transported into the coronary arteries. At this arterial limb of the circuit either gadolinium contrast agent or microspheres can be directly injected. The venous blood is collected in the heart chamber after passive ejection of the right ventricle and recirculated in the system.

perfusion analysis. On the subsegmental level, 30 voxels were included in the subendocardial and subepicardial and 40 voxels in the midmyocardial subsegments, respectively. For the purpose of this paper, the AHA segments were called transmural segments. Prior to deconvolution analysis, baseline correction, spatial and temporal filtering and homogeneity correction were performed on the extracted SI curves. ${ }^{17}$

\section{Quantitative microsphere analysis using an imaging cryomicrotome}

Immediately after the gadobutrol injection, a total of 100000 microspheres were injected into the circulation at the same site used for gadolinium injection. Three different colours of microspheres were used during the experiments. Quantitative analysis of the microtome images was performed in similar slices used for perfusion quantification according to previously described methods. ${ }^{20,27}$ To assess microsphere deposition, cryomicrotome and CMR images were rigidly registered to the same co-ordinate space and microsphere flow quantified in each region of interest $(\mathrm{ROI})$ as a function of $\mathrm{ROI}$ volume, arterial flow rate, and microsphere count fraction (Figure 3). ${ }^{20}$ The cryomicrotome images effectively provided a point cloud of microspheres. This point cloud was aligned with the heart geometry in the MR images via image registration. An initial registration was achieved by identifying anatomical landmarks including the aortic valve, the LV apex, and the proximal LAD in the CMR and microtome images. In a second step, fine manual rigid transformation adjustments were performed using the $3 \mathrm{D}$ visualization software CMGUI (OpenCMISS Continuum Mechanics, Imaging, Signal processing and System identification; http://www.cmiss.org/cmgui). Special attention was paid to exactly align the upper and lower RV insertion points to precisely overlay the perfusion and cryomicrotome images. Myocardial segments for flow quantification were then defined as described above.

Segmental flow was calculated in units of $\mathrm{mL} / \mathrm{min} / \mathrm{g}$ of tissue from the following equation:

$$
\frac{N_{S}}{M_{S}} \times \frac{F_{t}}{N_{t}}
$$

where $N_{s}$ is the number of microspheres counted in a segment, $N_{t}$ is the total number of microspheres injected, $F_{\mathrm{t}}$ is the total arterial input flow rate in $\mathrm{mL} / \mathrm{min}$, and $M_{s}$ is the mass of a segment in grams. Mass was calculated from segment volume using the specific density of myocardial tissue $(1.05 \mathrm{~g} / \mathrm{mL})$.

\section{Signal-to-noise ratio}

Signal-to-noise ratios (SNRs) for each perfusion sequence and both field strengths were calculated by dividing each mean signal intensity (SI) by the standard deviation (SD) of the background noise (measured in the air around the heart). 


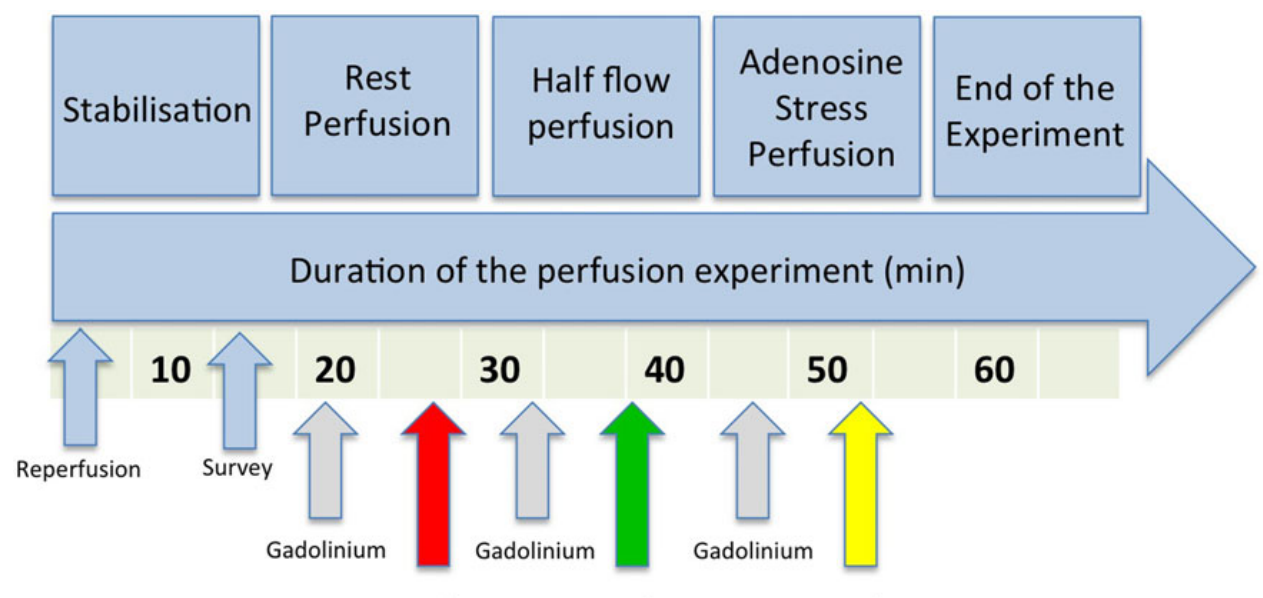

Microspheres Microspheres Microspheres

Figure 2 Flow chart of the time course of the experiments. After preparation stability was achieved, CMR imaging was started with the acquisition of rest perfusion images. Microspheres were injected after the gadolinium injection for perfusion imaging. This process was repeated with $50 \%$ of the flow and during adenosine-mediated pharmacological vasodilation in a constant pressure perfusion mode. The order of the changes made to the flow and the respective colour of microspheres with each injection were randomized.

\section{Statistics}

Data analysis was performed with IBM SPSS statistics for Mac 20.0.0 (SPSS Inc., Chicago, IL, USA). Continuous data are expressed as the mean \pm $\mathrm{SD}$. The paired sample $t$-test was used to compare perfusion measurements at rest and with stress and at low flow with the respective microsphere flow values within each heart. To assess agreement between the CMR perfusion measurements and microspheres, we used linear regression analysis and the method proposed by Bland and Altman. ${ }^{28}$ The Fisher $z$ transformation was used to compare the correlation coefficients of the quantitative analysis and microspheres between field strengths and spatial location. A $P$-value of $<0.05$ was considered statistically significant using comparisons on a per animal base. To account for repeated measurements in the same animals, $P$-values for repeated segmental comparisons are reported for descriptive purposes and not for the indication of statistical significance.

\section{Results}

All hearts recovered electrical function upon reperfusion. Hearts were defibrillated for $3 \pm 2$ times on average. All hearts remained stable throughout the perfusion experiments. No heart required additional defibrillation after the stabilization phase. Heart rate was $73 \pm 17$ bpm. Heart weight was $269 \pm 20 \mathrm{~g}$. The external roller pump baseline blood flow was set to $245 \pm 40 \mathrm{~mL} / \mathrm{min}$. Further measurements were taken during reduced flow at $123 \pm 18 \mathrm{~mL} / \mathrm{min}$ and during adenosine-induced hyperaemia with $401 \pm 43 \mathrm{~mL} / \mathrm{min}$. These flow rates constituted an overall LV flow of $0.91 \pm 0.17$, $0.47 \pm 0.09$, and $1.52 \pm 0.1 \mathrm{~mL} / \mathrm{min} / \mathrm{g}$, respectively.

High-resolution voxel-wise assessment of perfusion was able to distinguish between occluded and remote myocardium for all flow conditions $(P<0.001)$. Furthermore, perfusion estimates in the normally perfused areas at rest was distinguished from perfusion during half flow and hyperaemic perfusion at $1.5 \mathrm{~T}$
$(1.22 \pm 0.92 \mathrm{~mL} / \mathrm{min} / \mathrm{g}, \quad 0.56 \pm 0.37 \mathrm{~mL} / \mathrm{min} / \mathrm{g}$, and $2.21 \pm 1.67$ $\mathrm{mL} / \mathrm{min} / \mathrm{g}$, respectively, $P<0.001)$ and at $3 \mathrm{~T}(0.99 \pm 0.31 \mathrm{~mL} / \mathrm{min} / \mathrm{g}$, $0.55 \pm 0.26 \mathrm{~mL} / \mathrm{min} / \mathrm{g}$, and $2.08 \pm 0.81 \mathrm{~mL} / \mathrm{min} / \mathrm{g}$, respectively, $P<0.001$; Figure 4).

Figures 5 and 6 and Table 1 show the relation between microspheres and CMR-derived perfusion estimates at both field strengths. There was an excellent correlation at both field strengths: $3 \mathrm{~T}(r=0.96 ; P<0.001)$ and $1.5 \mathrm{~T}(r=0.94 ; P<0.001)$. The mean bias between CMR perfusion and microspheres was minimal both at $1.5 \mathrm{~T}(0.03 \pm 0.35 \mathrm{~mL} / \mathrm{min} / \mathrm{g})$ and at $3 \mathrm{~T}(0.07 \pm 0.23 \mathrm{~mL} / \mathrm{min} / \mathrm{g})$. The analysis after subdivision of the segments into different layers revealed slightly higher perfusion values within the subendocardium and the midmyocardium as opposed to the subepicardium measured with both CMR perfusion and microspheres (Table 2). There was also good agreement at both field strengths (Table 1, Figures 5 and 6). While subendocardial perfusion estimates showed the lowest bias at both field strengths $(0.02 \pm 0.48 \mathrm{~mL} / \mathrm{min} / \mathrm{g}$ at $1.5 \mathrm{~T}$ and $-0.02 \pm$ $0.45 \mathrm{~mL} / \mathrm{min} / \mathrm{g}$ at $3 \mathrm{~T})$, midmyocardial perfusion $(-0.08 \pm 0.57 \mathrm{~mL}$ $\mathrm{min} / \mathrm{g}$ at $1.5 \mathrm{~T}$ and $0.05 \pm 0.28 \mathrm{~mL} / \mathrm{min} / \mathrm{g}$ at $3 \mathrm{~T})$ and subepicardial perfusion $(0.06 \pm 0.37 \mathrm{~mL} / \mathrm{min} / \mathrm{g}$ at $1.5 \mathrm{~T}$ and $0.17 \pm 0.42 \mathrm{~mL} / \mathrm{min} / \mathrm{g}$ at $3 \mathrm{~T})$ were associated with slightly higher bias.

There was no significant difference in correlation strength between high-resolution voxel-wise quantitative perfusion estimates and microspheres between 1.5 and $3 \mathrm{~T}$ at the transmural, subendocardial, midmyocardial, and subepicardial level. However, there was a trend towards better correlation at the midmyocardial level at $3 \mathrm{~T}(P=0.03)$.

Bland Altman analysis showed good agreement between CMR-derived quantitative perfusion estimates and the microsphere reference standard for transmural segments as well as for different myocardial layers as shown in Figures 5 and 6 and Table 2. The 95\% confidence intervals $(\mathrm{Cl})$ were slightly narrower at $3 \mathrm{~T}$ compared with $1.5 \mathrm{~T}$ (Table 2). 


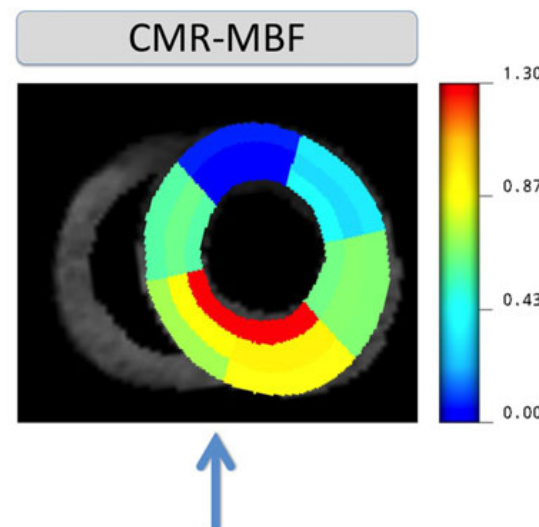

Segmentation
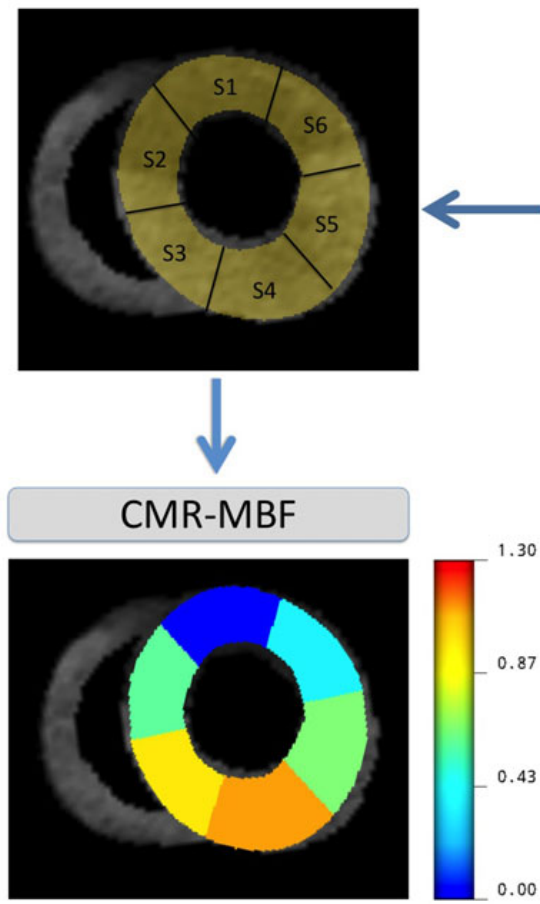

\section{Microsphere-MBF}

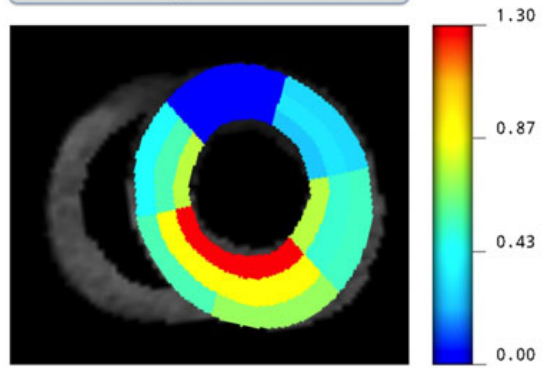

A
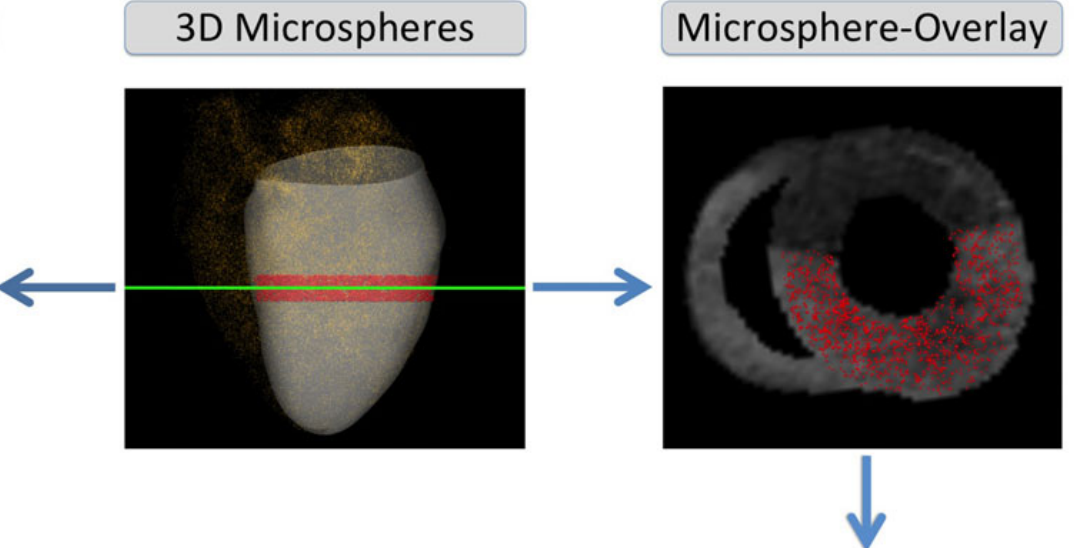

Microsphere-MBF

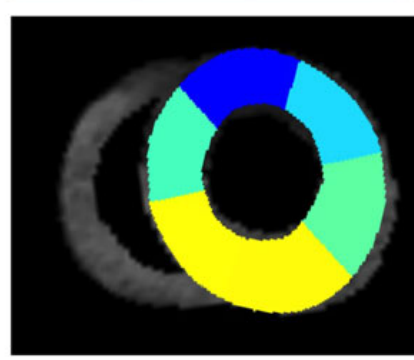
1.30

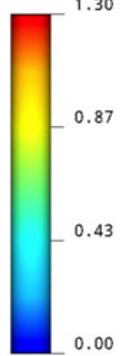

Figure 3 The figure shows an example of a rest perfusion study after selective occlusion of the LAD at 3 T. The centre image displays a 3D representation of all microspheres after rigid registration of the cryomicrotome images with the CMR images. The image on the right of the middle row shows the perfusion CMR image overlaid with the microspheres (red dots). Segmental microsphere quantification reveals the regional perfusion defect as a consequence to the LAD ligation and the normal perfusion in remote myocardium (lower right corner). A more detailed relationship of the regional flow patterns can be appreciated from the quantitative microsphere images subdivided into subendocardial, midmyocardial, and subendocardial layers (upper right corner). In the middle row, the segmentation of the perfusion image is displayed (middle left). On the lower left, the segmental quantitative perfusion values based on averaged voxel-wise Fermi function-constrained deconvolution is shown. The upper left shows the same images with quantitative values for subendocardial, midmyocardial, and subepicardial layers. Looking at the voxel-wise SI curves for Fermi function-constrained deconvolution in the same area delivers quantitative CMR-based results $[\mathrm{mL} / \mathrm{min} / \mathrm{g}]$ that match the microsphere-based results. Please note CMR images have been segmented to increase visibility.

The quantification error for CMR perfusion compared with microspheres increased slightly with higher perfusion rates (Table 3).

\section{Signal-to-noise ratio}

The SNR values for the perfusion sequences were $26 \pm 13$ and $10.9 \pm 3.9$ at 3 and $1.5 \mathrm{~T}$, respectively.

\section{Discussion}

This work has several notable findings. First, it demonstrates that Fermi function-constrained deconvolution quantitative voxel-wise MR perfusion analysis accurately estimates MBF using state of the art MR perfusion acquisitions. Secondly, it demonstrates that the 


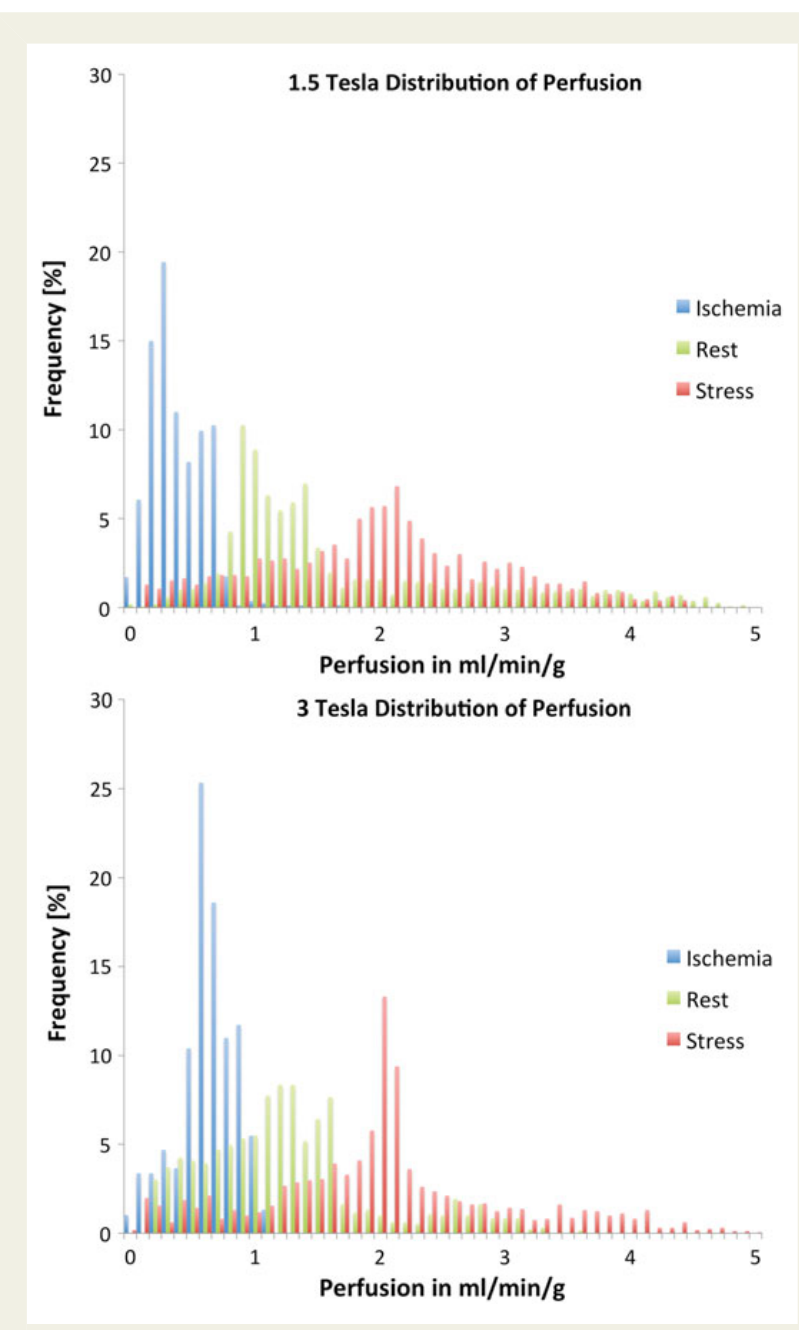

Figure 4 Histograms of estimated MBF comparing the distribution of perfusion values obtained by voxel-wise analysis during rest perfusion, half flow representing 'ischaemia' and adenosine-induced hyperaemia representing 'stress' at $1.5 \mathrm{~T}$ (top row) and $3 \mathrm{~T}$ (bottom row). Values are displayed for the normally perfused myocardium.

spatial resolution of voxel-wise analysis can be utilized to assess MBF in different myocardial layers. Third, this was equally feasible at 3 and 1.5 T despite the differences in spatial resolution and SNR.

We have chosen the Fermi function-based deconvolution algorithm in the current work. Recent validation work performed using isolated blood-perfused pig heart and synthetic perfusion phantom experiments have demonstrated very accurate performance and low sensitivity to noise of Fermi function-based deconvolution at the segmental level in comparison with other quantitative algorithms such as autoregressive moving average modelling, exponential basis deconvolution, and B-spline basis deconvolution. ${ }^{17,27}$ While the SNR levels at both field strengths in the experimental setup would also accommodate for the use of other deconvolution algorithms, in the clinical environment lower SNR levels are found especially with the high-resolution techniques, irrespective of field strength. Furthermore, the utility of Fermi-based quantification has been repeatedly shown in different clinical studies. ${ }^{29-31}$ The utilization of a controlled environment where MBF to the heart is known and its distribution over time within the myocardium quantified with 3D microspheres now allows a highly accurate validation of Fermi-based CMR perfusion at the voxel level. ${ }^{20,21}$ In comparison to previous methodology, the microsphere methodology we have employed based on 3D datasets obtained by cryomicrotome cuts and images may provide superior accuracy, because it does not require to physically dissect the heart into segments corresponding to those used for CMR perfusion quantification ${ }^{11,18,32}$ but provides accurate anatomical alignment of the heart geometry for the comparison of microspheres and CMR perfusion.

Recently, Motwani et al. ${ }^{15}$ suggested that higher spatial resolutions allow improved visual detection of subendocardial ischaemia. In the current study, the subendocardial quantitative perfusion assessed with CMR showed excellent correlation with microspheres both at 1.5 and $3 \mathrm{~T}$, suggesting that the findings reported by Motwani et al. may be quantitatively objectified using the methodology proposed in the current paper.

The correlation strength of CMR perfusion and microspheres did not reach significant statistical difference between field strengths or analysis levels, even though in theory the superior spatial resolution and higher SNR at 3 T may facilitate voxel-wise perfusion quantification.

The current work demonstrates the feasibility of the voxel-wise technique for both field strengths regardless of spatial resolution. Voxel-wise perfusion imaging had initially been described and validated in non-beating isolated perfused pig hearts at $1.5 \mathrm{~T}^{33}$ Subsequently, Zarinabad et al. ${ }^{17}$ have demonstrated its feasibility in a MR compatible perfusion phantom ${ }^{34}$ and in humans at $3 \mathrm{~T}$. Further in vivo validation work has been published by Hsu et al., ${ }^{18}$ who demonstrated that voxelwise quantification can be utilized at $1.5 \mathrm{~T}$ to determine differences in flow between subepicardium and subendocardium. They furthermore demonstrated a very accurate assessment of quantitative perfusion in canines compared with microspheres. The present study adds to the literature in several aspects. First, it adds the in vivo validation against microspheres to the recent publication of Zarinabad et al. that demonstrated the feasibility of quantitative voxel-wise perfusion imaging but was not able to confirm the findings with a histological reference standard as phantom and patient studies were involved. ${ }^{17}$ Secondly, it shows the feasibility and compares the performance of voxel-wise quantitative CMR perfusion in a very well-controlled animal model at 1.5 and $3 \mathrm{~T}$, demonstrating its value at both field strengths. The demonstration of quantitative myocardial voxel-wise perfusion imaging now shows the feasibility of high-resolution techniques in the isolated heart model, which has previously been exposed to segmental quantification only. ${ }^{27}$ Lastly in our study, we were able to confirm the good agreement between voxel-wise quantitative perfusion imaging and microspheres at $1.5 \mathrm{~T}$ as described by Hsu et al. ${ }^{18}$ The applicability of imaging and quantification techniques to both commonly used clinical field strengths is important and in line with recent evidence from our group that demonstrates that standard kt-BTFE-based CMR perfusion imaging at 1.5 $\mathrm{T}$ has similar diagnostic accuracy for quantitative myocardial perfusion reserve analysis as the clinical reference standard of quantification positron emission tomography (PET) in patients with CAD. ${ }^{30}$ Most clinical CMR examinations are performed at $1.5 \mathrm{~T}$, and it is therefore crucial to have a working methodology for this scenario. The sequence we have used at $1.5 \mathrm{~T}$ is identical to the perfusion sequence used in a major ongoing CMR perfusion clinical trial 

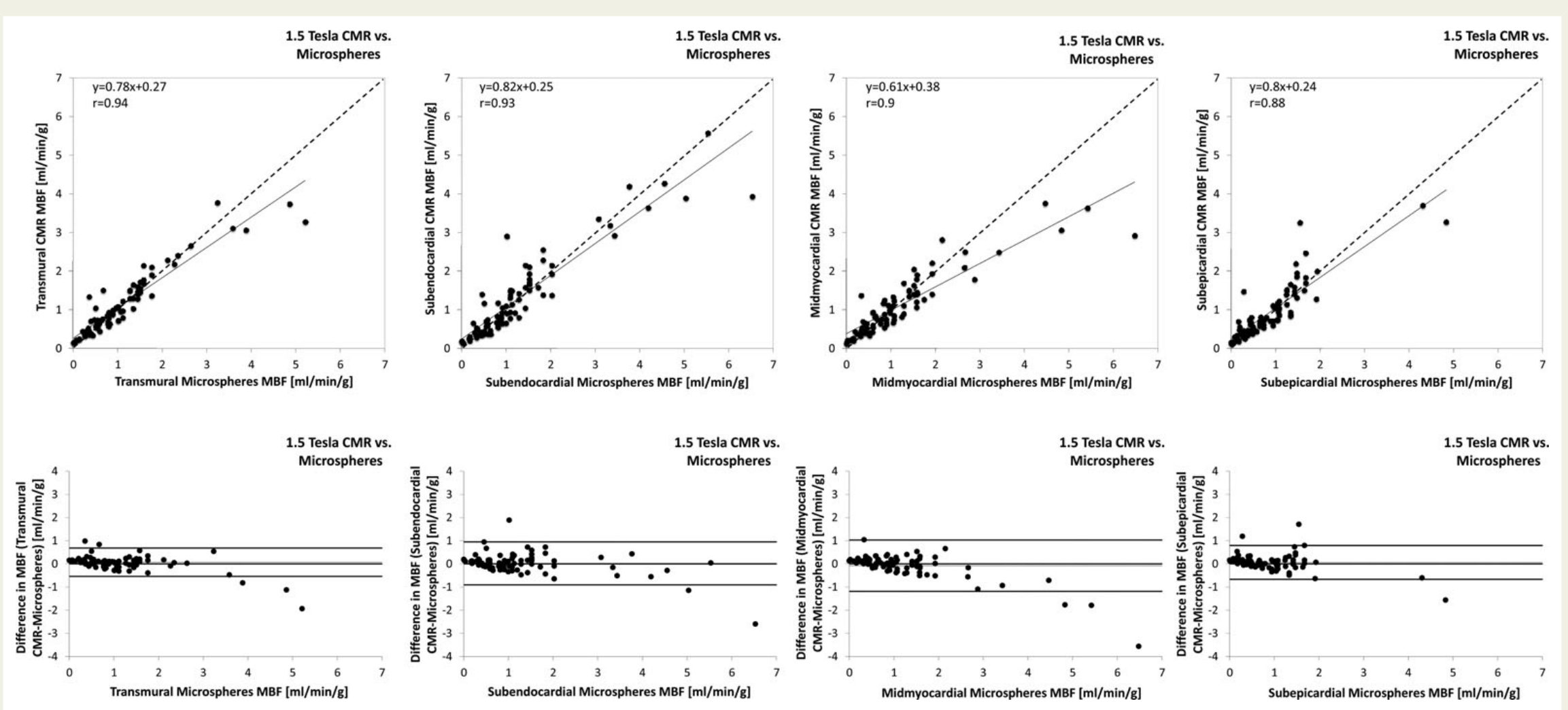

Figure 5 The figure shows the correlation of CMR-derived quantitative perfusion analysis with the reference standard of microsphere-derived quantitative perfusion at $1.5 \mathrm{~T}$ (top row). The bottom row shows the agreement between CMR-derived perfusion and the reference standard using the Bland Altman analysis. Results are shown for AHA standard transmural segments and subsegments (subendocardial, midmyocardial, and subepicardial) from left to right. 

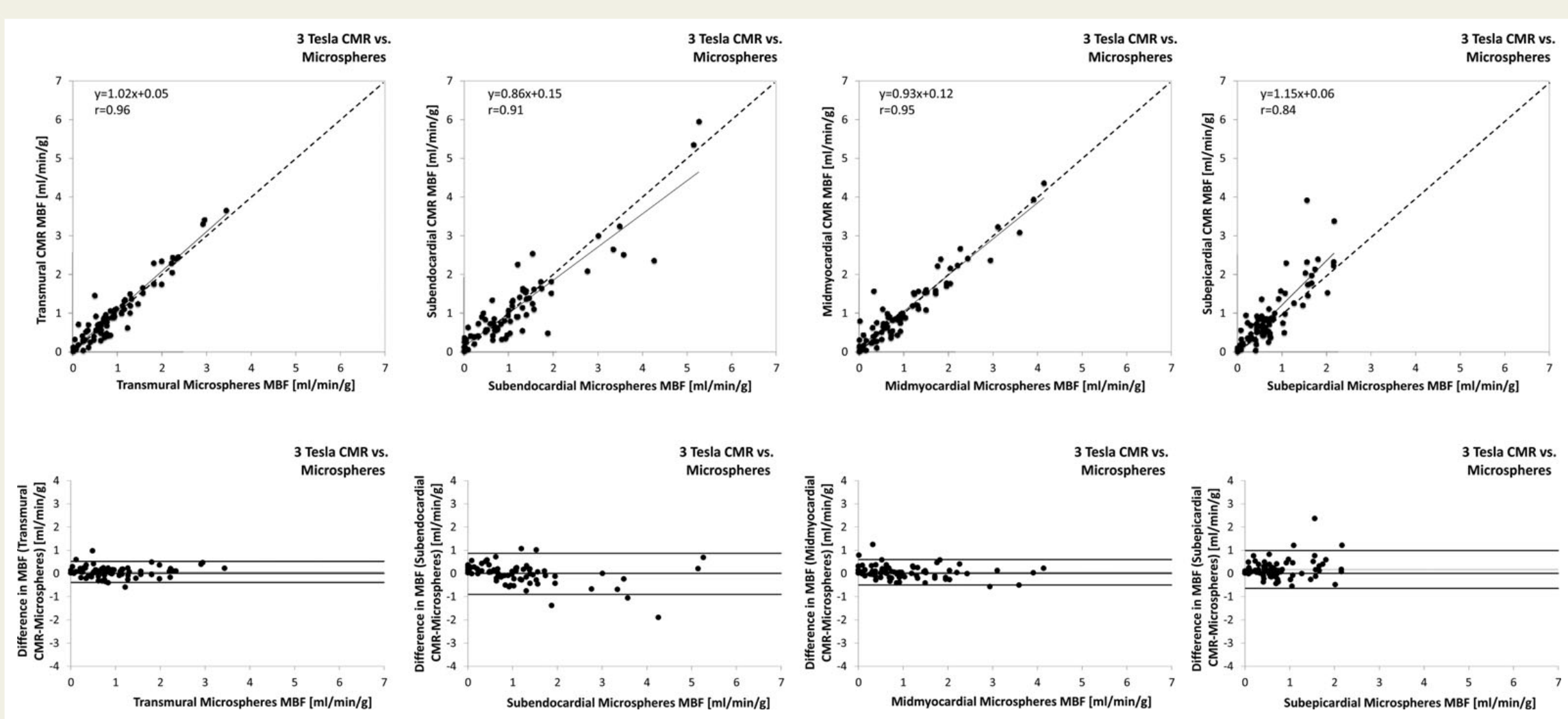

Figure 6 The figure shows the correlation of CMR-derived quantitative perfusion analysis with the reference standard of microsphere-derived quantitative perfusion at $3 \mathrm{~T}$ (top row). The bottom row shows the agreement between CMR-derived perfusion and the gold standard using the Bland Altman analysis. Results are shown for AHA standard transmural segments and subsegments (subendocardial, midmyocardial, and subepicardial) from left to right. 
Table I Correlation of CMR MBF at the different field strengths and spatial locations with the gold standard microspheres

\begin{tabular}{|c|c|c|c|c|c|}
\hline \multirow[t]{2}{*}{ Spatial location } & \multicolumn{2}{|c|}{$1.5 \mathrm{~T}$} & \multicolumn{2}{|l|}{$3 T$} & \multirow[t]{2}{*}{$P$-value } \\
\hline & $r$ & $y$ & $r$ & $y$ & \\
\hline Transmural & 0.94 & $0.78 x+0.27$ & 0.96 & $1.02 x+0.05$ & $<0.001$ \\
\hline Subendocardial & 0.93 & $0.82 x+0.25$ & 0.91 & $0.86 x+0.15$ & $<0.001$ \\
\hline Midmyocardial & 0.9 & $0.61 x+0.38$ & 0.95 & $0.93 x+0.12$ & $<0.001$ \\
\hline Subepicardial & 0.88 & $0.8 x+0.24$ & 0.84 & $1.15 x+0.06$ & $<0.001$ \\
\hline
\end{tabular}

The table shows the correlation coefficients $(r)$, and the $P$-values indicating the correlation strength as well as the $y$ intercepts.

Table 2 Mean perfusion values $(\mathrm{mL} / \mathrm{min} / \mathrm{g})$ based on microspheres and CMR for both field strengths

\begin{tabular}{|c|c|c|c|c|c|}
\hline Field strength & Spatial location & Modality & Mean (mL/min/g) & Mean difference and $\mathrm{Cl}(95 \%)$ of the difference & $P$-value \\
\hline \multirow[t]{8}{*}{$1.5 \mathrm{~T}$} & \multirow[t]{2}{*}{ Transmural } & CMR & $1.1 \pm 0.82$ & \multirow[t]{2}{*}{$0.03(-0.04$ to 0.11$)$} & \multirow[t]{2}{*}{0.4} \\
\hline & & Microspheres & $1.07 \pm 0.98$ & & \\
\hline & \multirow[t]{2}{*}{ Subendocardial } & CMR & $1.28 \pm 1.11$ & \multirow[t]{2}{*}{$0.02(-0.08$ to 0.13$)$} & \multirow[t]{2}{*}{0.65} \\
\hline & & Microspheres & $1.25 \pm 1.25$ & & \\
\hline & \multirow[t]{2}{*}{ Midmyocardial } & CMR & $1.08 \pm 0.78$ & \multirow[t]{2}{*}{$-0.08(-0.2$ to 0.05$)$} & \multirow[t]{2}{*}{0.21} \\
\hline & & Microspheres & $1.16 \pm 1.16$ & & \\
\hline & \multirow[t]{2}{*}{ Subepicardial } & CMR & $0.94 \pm 0.71$ & \multirow[t]{2}{*}{$0.06(-0.02$ to 0.15$)$} & \multirow[t]{2}{*}{0.12} \\
\hline & & Microspheres & $0.88 \pm 0.77$ & & \\
\hline \multirow[t]{8}{*}{$3 \mathrm{~T}$} & \multirow[t]{2}{*}{ Transmural } & CMR & $1 \pm 0.79$ & \multirow[t]{2}{*}{0.07 (0.02 to 0.12$)$} & \multirow[t]{2}{*}{0.1} \\
\hline & & Microspheres & $0.94 \pm 0.75$ & & \\
\hline & \multirow[t]{2}{*}{ Subendocardial } & CMR & $1.11 \pm 1.04$ & \multirow[t]{2}{*}{$-0.02(-0.12$ to 0.09$)$} & \multirow[t]{2}{*}{0.75} \\
\hline & & Microspheres & $1.12 \pm 1.11$ & & \\
\hline & \multirow[t]{2}{*}{ Midmyocardial } & CMR & $1.08 \pm 0.89$ & \multirow[t]{2}{*}{$0.05(-0.01$ to 0.12$)$} & \multirow[t]{2}{*}{0.12} \\
\hline & & Microspheres & $1.03 \pm 0.9$ & & \\
\hline & \multirow[t]{2}{*}{ Subepicardial } & CMR & $0.93 \pm 0.76$ & \multirow[t]{2}{*}{$0.17(0.08-0.27)$} & \multirow[t]{2}{*}{$<0.001$} \\
\hline & & Microspheres & $0.75 \pm 0.56$ & & \\
\hline
\end{tabular}

Data are presented as mean \pm standard deviation (SD) along with $P$-values based on the paired $t$-test.

$\mathrm{Cl}$, confidence intervals; CMR, cardiac magnetic resonance.

Table 3 Mean perfusion values based on microspheres and CMR for both field strengths for microsphere perfusion values $\geq 2(\mathrm{~mL} / \mathrm{min} / \mathrm{g})$

\begin{tabular}{|c|c|c|c|c|c|}
\hline Field strength & Spatial location & Modality & Mean & Mean difference and $\mathrm{Cl}(95 \%)$ of the difference & $P$-value \\
\hline \multirow[t]{4}{*}{$1.5 \mathrm{~T}$} & Transmural & $\begin{array}{l}\text { CMR } \\
\text { Microspheres }\end{array}$ & $\begin{array}{l}2.95 \pm 0.6 \\
3.35 \pm 1.14\end{array}$ & $-0.4(-0.99$ to 0.19$)$ & 0.16 \\
\hline & Subendocardial & $\begin{array}{l}\text { CMR } \\
\text { Microspheres }\end{array}$ & $\begin{array}{r}3.89 \pm 0.78 \\
4.4 \pm 1.15\end{array}$ & $-0.5(-1.2$ to 0.21$)$ & 0.14 \\
\hline & Midmyocardial & $\begin{array}{l}\text { CMR } \\
\text { Microspheres }\end{array}$ & $\begin{array}{c}2.78 \pm 0.65 \\
3.8 \pm 1.5\end{array}$ & $-1.1(-2.02$ to -0.18$)$ & 0.03 \\
\hline & Subepicardial & $\begin{array}{l}\text { CMR } \\
\text { Microspheres }\end{array}$ & $\begin{array}{l}2.16 \pm 1 \\
2.24 \pm 1.35\end{array}$ & $-0.08(-0.79$ to 0.63$)$ & 0.8 \\
\hline \multirow[t]{4}{*}{$3 \mathrm{~T}$} & Transmural & $\begin{array}{l}\text { CMR } \\
\text { Microspheres }\end{array}$ & $\begin{array}{l}2.76 \pm 0.61 \\
2.57 \pm 0.46\end{array}$ & $0.18(0.02$ to 0.35$)$ & 0.04 \\
\hline & Subendocardial & $\begin{array}{l}\text { CMR } \\
\text { Microspheres }\end{array}$ & $\begin{array}{r}3.4 \pm 1.45 \\
3.85 \pm 0.94\end{array}$ & $-0.45(-1.13$ to 0.22$)$ & 0.16 \\
\hline & Midmyocardial & $\begin{array}{l}\text { CMR } \\
\text { Microspheres }\end{array}$ & $\begin{array}{l}3.04 \pm 0.78 \\
3.02 \pm 0.84\end{array}$ & $0.03(-0.29$ to 0.35$)$ & 0.86 \\
\hline & Subepicardial & $\begin{array}{l}\text { CMR } \\
\text { Microspheres }\end{array}$ & $\begin{array}{l}2.38 \pm 0.87 \\
1.57 \pm 0.42\end{array}$ & $0.81(0.12$ to 1.5$)$ & 0.03 \\
\hline
\end{tabular}

Data are presented as mean \pm standard deviation (SD) along with $P$-values based on the paired $t$-test.

$\mathrm{Cl}$, confidence intervals; CMR, cardiac magnetic resonance. 
(MR-INFORM, clinicaltrials.gov NCT01236807). ${ }^{35}$ Our study shows that voxel-wise quantitative perfusion should be possible in this trial.

There is accumulating evidence indicating the utility of quantitative perfusion analysis for various diseases to detect early and subtle changes. We believe that a robust assessment of blood flow alterations based on high-resolution voxel-wise quantification may expand the clinical applications of quantitative perfusion assessment and could add onto the diagnostic accuracy of the methodology.

\section{Limitations}

The sample size of the current study was relatively small but comparable with previously performed studies. ${ }^{18}$ The aim of the current study was the validation of MR perfusion in different segments and not a per-subject comparison. The fact that the explanted heart is less physiological allows for a highly controlled setup with minimal external influences such as heart rate and cardiac output. On the other hand, this model oversimplifies in vivo physiological conditions with complex nervous cardiac regulation and breathing motion during stress. The absence of first pass of contrast agent through the LV increases the contrast between myocardium and LV cavity in the current experimental setting which also represents a significant difference from in vivo imaging. Furthermore, there is also absence of offresonance influences from the lungs and diaphragm in the isolated heart. The current validation setup may be further improved by implementing a fully working heart setup and may also include an ordinal group of flow reductions, e.g. 30,60 , and $90 \%$ reductions to acquire even more validation data.

The current paper describes validation of voxel-wise quantification with CMR. We have compared the quantitative voxel-wise perfusion results to segments and myocardial layers. While a true voxel by voxel validation with CMR and a reference standard would be of interest, the flow heterogeneity inherent to the microsphere methodology prevents such validation studies. ${ }^{26}$

Future studies need to bring this promising technique to a clinical scenario and determine its diagnostic accuracy to detect functionally significant CAD and quantify ischaemic burden in patients.

\section{Conclusions}

CMR-derived voxel-wise quantitative blood flow estimates very accurately resemble true MBF in a controlled animal model. This holds true irrespective of spatial location or field strength. Using state of the art perfusion sequences and quantification algorithms is essential to allow CMR perfusion to be quantified with high resolution at the voxel level, which has the potential to develop into a useful clinical tool.

\section{Conflict of interest: None declared.}

\section{Funding}

A.S. is a British Heart Foundation (BHF) Clinical Research Fellow (FS/10/ 029/28253) and received grant support from the BHF (RE/08/003) and the Biomedical Research Centre (BRC-CTF 196). M.S. receives support from the Engineering and Physical Sciences Research Council (EP/H046410/1). E.N. receives grant support from BHF (RE/08/003), the Wellcome Trust and Engineering and Physical Sciences Research Council (EPSRC, WT 088641/Z/09/Z), and the National Institute for
Health Research (NIHR) via the comprehensive BRC award to Guy's and St Thomas' NHS Foundation Trust in partnership with King's College London and King's College Hospital NHS Foundation Trust. A.C. is funded by the Wellcome Trust and EPSRC under grant number WT $088641 / Z / 09 / Z$. J.P.H.M.v.d.W. is funded by a VENI grant of the Netherlands Organization for Scientific Research (NWO/ZonMw 916.11.171). This study was further supported by grants to the AMC (J.A.E.S. and M.S.) from the Netherlands Heart Foundation (NHS 2006B186 and 2006B226), the Netherlands Organization for Health Research and Development (ZonMw 91105008 and 91112030), and the European Community (FP7-2007-224495: euHeart project). E.N. received significant grant support from Bayer Healthcare and Philips Healthcare. A.C. received grant support from Philips Healthcare.

\section{References}

1. Murray CJ, Lopez AD. Alternative projections of mortality and disability by cause 1990-2020: Global Burden of Disease Study. Lancet 1997;349:1498-504.

2. Hachamovitch R, Rozanski A, Hayes SW, Thomson LE, Germano G, Friedman JD et al. Predicting therapeutic benefit from myocardial revascularization procedures: are measurements of both resting left ventricular ejection fraction and stress-induced myocardial ischemia necessary? J Nucl Cardiol 2006;13:768-78.

3. Nagel E, Klein C, Paetsch I, Hettwer S, Schnackenburg B, Wegscheider K et al. Magnetic resonance perfusion measurements for the noninvasive detection of coronary artery disease. Circulation 2003;108:432-7.

4. Lee DC, Simonetti OP, Harris KR, Holly TA, Judd RM, Wu E et al. Magnetic resonance versus radionuclide pharmacological stress perfusion imaging for flow-limiting stenoses of varying severity. Circulation 2004;110:58-65.

5. Nagel E, Lehmkuhl HB, Bocksch W, Klein C, Vogel U, Frantz E et al. Noninvasive diagnosis of ischemia-induced wall motion abnormalities with the use of high-dose dobutamine stress MRI: comparison with dobutamine stress echocardiography. Circulation 1999;99:763-70.

6. Greenwood JP, Maredia N, Younger JF, Brown JM, Nixon J, Everett CC et al. Cardiovascular magnetic resonance and single-photon emission computed tomography for diagnosis of coronary heart disease (CE-MARC): a prospective trial. Lancet 2012; 379:453-60.

7. Morton G, Schuster A, Perera D, Nagel E. Cardiac magnetic resonance imaging to guide complex revascularization in stable coronary artery disease. Eur Heart J 2010;31:2209-15.

8. Plein S, Ryf S, Schwitter J, Radjenovic A, Boesiger P, Kozerke S. Dynamic contrast-enhanced myocardial perfusion MRI accelerated with k-t sense. Magn Reson Med 2007;58:777-85.

9. Ishida M, Morton G, Schuster A, Nagel E, Chiribiri A. Quantitative assessment of myocardial perfusion MRI. Curr Cardiovasc Imaging Rep 2010;3:65-73.

10. Morton G, Jogiya R, Plein S, Schuster A, Chiribiri A, Nagel E. Quantitative cardiovascular magnetic resonance perfusion imaging: inter-study reproducibility. Eur Heart J Cardiovasc Imaging 2012;13:954-60.

11. Christian TF, Rettmann DW, Aletras AH, Liao SL, Taylor JL, Balaban RS et al. Absolute myocardial perfusion in canines measured by using dual-bolus first-pass MR imaging. Radiology 2004;232:677-84.

12. Christian TF, Aletras AH, Arai AE. Estimation of absolute myocardial blood flow during first-pass MR perfusion imaging using a dual-bolus injection technique: comparison to single-bolus injection method. J Magn Reson Imaging 2008;27:1271-7.

13. Jerosch-Herold M, Swingen C, Seethamraju RT. Myocardial blood flow quantification with MRI by model-independent deconvolution. Med Phys 2002;29:886-97.

14. Cerqueira MD, Weissman NJ, Dilsizian V, Jacobs AK, Kaul S, Laskey WK et al. Standardized myocardial segmentation and nomenclature for tomographic imaging of the heart: a statement for healthcare professionals from the Cardiac Imaging Committee of the Council on Clinical Cardiology of the American Heart Association. Circulation 2002;105:539-42.

15. Motwani M, Maredia N, Fairbairn TA, Kozerke S, Radjenovic A, Greenwood JP et al. High-resolution versus standard-resolution cardiovascular MR myocardial perfusion imaging for the detection of coronary artery disease. Circ Cardiovasc Imaging 2012;5:306-13.

16. Motwani M, Lockie T, Greenwood JP, Plein S. Accelerated, high spatial resolution cardiovascular magnetic resonance myocardial perfusion imaging. I Nud Cardiol 2011;18:952-8.

17. Zarinabad N, Chiribiri A, Hautvast GLTF, Ishida M, Schuster A, Cvetkovic Z et al. Voxel-wise quantification of myocardial perfusion by cardiac magnetic resonance. Feasibility and methods comparison. Magn Reson Med 2012;68:1994-2004.

18. Hsu L-Y, Groves DW, Aletras AH, Kellman P, Arai AE. A quantitative pixel-wise measurement of myocardial blood flow by contrast-enhanced first-pass CMR 
perfusion imaging: microsphere validation in dogs and feasibility study in humans. JACC Cardiovasc Imaging 2012;5:154-66.

19. Schuster A, Grünwald I, Chiribiri A, Southworth R, Ishida M, Hay G et al. An isolated perfused pig heart model for the development, validation and translation of novel cardiovascular magnetic resonance techniques. J Cardiovasc Magn Reson 2010;12:53.

20. van Horssen P, Siebes M, Hoefer I, Spaan JAE, van den Wijngaard JPHM. Improved detection of fluorescently labeled microspheres and vessel architecture with an imaging cryomicrotome. Med Biol Eng Comput 2010;48:735-44.

21. Spaan JAE, Ter Wee R, van Teeffelen JWGE, Streekstra G, Siebes M, Kolyva C et al. Visualisation of intramural coronary vasculature by an imaging cryomicrotome suggests compartmentalisation of myocardial perfusion areas. Med Biol Eng Comput 2005;43:431-5.

22. Schuster A, Chiribiri A, Ishida M, Morton G, Paul M, Hussain ST et al. Cardiovascular magnetic resonance imaging of isolated perfused pig hearts in a $3 \mathrm{~T}$ clinical MR scanner. Interv Med Appl Sci 2012;4:186-92.

23. Ishida M, Schuster A, Morton G, Chiribiri A, Hussain S, Paul Met al. Development of a universal dual-bolus injection scheme for the quantitative assessment of myocardial perfusion cardiovascular magnetic resonance. J Cardiovasc Magn Reson 2011;13:28.

24. Hautvast G, Chiribiri A, Zarinabad N, Schuster A, Breeuwer M, Nagel E. Myocardial blood flow quantification from MRI by deconvolution using an exponential approximation basis. IEEE Trans Biomed Eng 2012;59:2060-7.

25. Jerosch-Herold M, Seethamraju RT, Swingen CM, WilkeNM, Stillman AE. Analysis of myocardial perfusion MRI. J Magn Reson Imaging 2004;19:758-70.

26. Decking UK, Pai VM, Bennett E, Taylor JL, Fingas CD, Zanger K et al. High-resolution imaging reveals a limit in spatial resolution of blood flow measurements by microspheres. Am J Physiol Heart Circ Physiol 2004;287:H1132-40.

27. Schuster A, Zarinabad N, Ishida M, Sinclair M, van den Wijngaard JP, Morton G et al. Quantitative assessment of magnetic resonance derived myocardial perfusion measurements using advanced techniques: microsphere validation in an explanted pig heart system. J Cardiovasc Magn Reson 2014;16:82

28. Bland JM, Altman DG. Statistical methods for assessing agreement between two methods of clinical measurement. Lancet 1986:1:307-10.

29. Lockie T, Ishida M, Perera D, Chiribiri A, De Silva K, Kozerke S et al. High-resolution magnetic resonance myocardial perfusion imaging at 3.0-tesla to detect hemodynamically significant coronary stenoses as determined by fractional flow reserve. J Am Coll Cardiol 2010;57:70-5.

30. Morton G, Chiribiri A, Ishida M, Hussain ST, Schuster A, Indermuehle A et al. Quantification of absolute myocardial perfusion in patients with coronary artery disease: comparison between cardiovascular magnetic resonance and positron emission tomography. J Am Coll Cardiol 2012;60:1546-55.

31. Patel AR, Antkowiak PF, Nandalur KR, West AM, Salerno M, Arora V et al. Assessment of advanced coronary artery disease advantages of quantitative cardiac magnetic resonance perfusion analysis. J Am Coll Cardiol 2010;56:561-9.

32. Christian TF, Bell SP, Whitesell L, Jerosch-Herold M. Accuracy of cardiac magnetic resonance of absolute myocardial blood flow with a high-field system: comparison with conventional field strength. JACC Cardiovasc Imaging 2009;2:1103-10.

33. Neyran B, Janier MF, Casali C, Revel D, Canet Soulas EP. Mapping myocardial perfusion with an intravascular MR contrast agent: robustness of deconvolution methods at various blood flows. Magn Reson Med 2002;48:166-79.

34. Chiribiri A, Schuster A, Ishida M, Hautvast G, Zarinabad N, Morton G et al. Perfusion phantom: An efficient and reproducible method to simulate myocardial first-pass perfusion measurements with cardiovascular magnetic resonance. Magn Reson Med 2013;69:698-707.

35. Hussain ST, Paul M, Plein S, Shah AM, McCann G, Marber MS et al. Design and rationale of the MR-INFORM study: stress perfusion cardiovascular magnetic resonance imaging to guide the management of patients with stable coronary artery disease. J Cardiovasc Magn Reson 2012;14:65. 